Trauma Berufskrankh 2015 • [Suppl 2]:

17:275-280

DOI 10.1007/s10039-015-0006-8

Online publiziert: 24. März 2015

๑) Springer-Verlag Berlin Heidelberg 2015

Claudia Drechsel-Schlund ${ }^{1} \cdot$ M. Weiß ${ }^{2} \cdot$ C. Krahl ${ }^{3} \cdot$ K. Romer-Raschidi ${ }^{2}$ B. Gruner ${ }^{4}$. H. Freytag ${ }^{5} \cdot$ R. Hoffmann ${ }^{6}$

${ }^{1}$ Berufsgenossenschaft für Gesundheitsdienst und Wohlfahrtspflege,

Bezirksverwaltung Würzburg, Würzburg, Deutschland

${ }^{2}$ Berufsgenossenschaftliche Unfallklinik Ludwigshafen, Abteilung für Psychologie, Ludwigshafen, Deutschland

${ }^{3}$ Berufsgenossenschaftliche Unfallklinik Frankfurt am Main,

Psychotraumatologisches Zentrum für Diagnostik und Therapie, Frankfurt, Deutschland

${ }^{4}$ Psychosomatische Praxis, Weimar, Deutschland

${ }^{5}$ Beufsgenossenschaftliche Unfallklinik Frankfurt am Main,

Psychotraumatologisches Zentrum für Diagnostik und Therapie, Frankfurt, Deutschland

${ }^{6}$ Berufsgenossenschaftliche Unfallklinik Frankfurt am Main,

Abteilung für Unfallchirurgie und Orthopädische Chirurgie, Frankfurt, Deutschland

\title{
Umsetzung des Psychotherapeutenverfahrens
}

\section{Einbindung von ärztlichen und psychologischen Psychotherapeuten}

Gewalterfahrungen am Arbeitsplatz sind Zugbegleiter, Rettungskräfte, Verkaufspersonal, Krankenschwestern und Pfleger, Taxifahrer, Rettungskräfte, Wach- und Sicherheitsleute, Dachdecker (• Abb. 1), Polizisten und weitere Beschäftigtengruppen mit intensivem Kontakt zu Kunden, Patienten, betreuten Personen oder Klienten.

Ebenso können sich psychische Störungen bei Arbeitsunfällen mit gravierenden körperlichen Verletzungen entwickeln, z. B. infolge eines lebensbedrohlichen Polytraumas. Zudem können Beschäftigte aller Branchen von belastenden Ereignissen am Arbeitsplatz betroffen sein, wenn sie als Ersthelfer bei einem schweren Unfall tätig oder Zeuge eines solchen werden, z. B. bei einem Brandunglück. Von besonderer Bedeutung in der Häufigkeit des Auftretens psychischer
Störungen sind zudem Wegeunfälle mit dem Fahrzeug.

\section{Hauptdiagnosen im Psychotherapeutenverfahren}

Auch bei Ereignissen mit hohem psychischen Traumatisierungspotenzial treten bei der Mehrzahl der Betroffenen keine überdauernden gesundheitlichen Folgen auf [1]. Bei einigen Personen entwickeln sich hingegen ereignisnah psychische Symptome, die eine frühzeitige professionelle psychotherapeutische Versorgung erfordern, um Komplikationen bzw. eine Chronifizierung zu verhindern [2]. Diese Fälle gilt es frühzeitig zu identifizieren. Zum Spektrum der psychischen Störungen nach Arbeitsunfällen zählen v. a. die akute Belastungsreaktion, Anpassungsstörungen, spezifische Phobie, posttrau-
In der GUV sind psychische Gesu heitsschäden ebenso wie körperliche Verletzungen vom Versicherungsschutz nach $\$ 8$ SGB VII umfasst. Psychische Unfallfolgen können nach ganz unterschiedlichen Unfallereignissen auftreten. Von medialem Interesse sind insbesondere Gewalt und Aggression am Arbeitsplatz, wie jüngst wieder ein tödlicher Messerangriff auf einen Mitarbeiter im Jobcenter Rothenburg ob der Tauber (03.12.2014). Besonders gefährdet für Bedrohungs- und
Ereignis: Sturz eines 36-jährigen Dachdeckers aus 4 Meter Höhe. Bewusstlosigkeit. Fraktur im Bereich der Schädelbasis, des HWK 2 und Kleinhirnblutung. Amnesie für das Unfallgeschehen. Diagnosen: Spezifische Phobie (Höhe), depressive Episode

Therapeutische Interventionen: Aufbau von Aktivitäten durch Vermittlung eines Krankheitsmodells, Aktivitätssteigerung durch Tagesstrukturierung. Unterstützung der beruflichen Neuorientierung. Begleitung der Umschulung. Konfrontationsbehandlung der Höhenangst. Ergebnis: Der Patient konnte die Umschulung abschließen und in dem neuen Beruf arbeiten. Fazit: Sehr gute Bilanz bei fortlaufender Abstimmung aller beteiligten Therapeuten und des Reha-Managers.

Abb. $1 \Delta$ Fallbeispiel 


\begin{tabular}{|l}
\hline Infobox Mehr Informationen \\
zum Thema \\
\hline Weitere Informationen sind unter folgenden \\
Links zu finden: \\
- www.dguv.de/landesverbaende/de/ \\
med_reha/Psychotherapeutenverfahren/ \\
index.jsp \\
- http://lviweb.dguv.de/dguvLviWeb/ \\
faces/p?_afrLoop=3622417865022000\&_ \\
afrWindowMode=0\&_adf.ctrl-sta- \\
te=1b9vg5hf84_4 \\
www.dguv.de/medien/formtexte/aerz- \\
te/F_2100/F2100.pdf
\end{tabular}

matische Belastungsstörungen, Angststörungen und depressive Episoden [3] sowie anhaltende Schmerzstörungen in Verbindung von körperlichen und psychischen Faktoren, die in kritischer Interaktion stehen können.

Im Folgenden werden relevante psychische Störungsbilder vorgestellt, die im Kontext der Versorgung von Patienten der Deutschen Gesetzlichen Unfallversicherung (DGUV) vornehmlich auftreten können [12]. Die Beschreibung der ausgewählten Störungsbilder bezieht sich auf die diagnostischen Kriterien der Internationalen statistischen Klassifikation der Krankheiten und verwandter Gesundheitsprobleme (ICD-10) der Weltgesundheitsorganisation (WHO; [7]).

Akute Belastungsreaktion. Die akute Belastungsreaktion wird als akute Reaktion mit beträchtlichem Schweregrad auf eine außergewöhnliche körperliche oder seelische Belastung bezeichnet. Es muss ein unmittelbarer und klarer zeitlicher Zusammenhang zwischen einer ungewöhnlichen Belastung und dem Beginn der Symptomatik vorliegen. Charakteristisch ist ein gemischtes und wechselhaftes Bild: anfänglich ein Zustand der Betäubung und Desorientiertheit, dann Depression, Angst, Ärger, Verzweiflung, Überaktivität und Rückzug bis hin zum dissoziativen Stupor. Vegetative Symptome von panischer Angst wie Tachykardie, Schwitzen und Atemnot können auftreten. Die Störung ist insgesamt rasch rückläufig und klingt nach Stunden bis einigen Tagen $a b$.

Anpassungsstörung. Besondere Lebensereignisse, auch gravierende Ereignisse oder Lebensveränderungen lösen häufig
Stresserleben mit emotionalen, kognitiven, physiologischen und verhaltensbezogenen Rektionen aus. Sie können eine notwendige Voraussetzung für die Verarbeitung dieser Ereignisse und Veränderungen sein. Geht die Auseinandersetzung mit der Belastungssituation und Lebensveränderung über eine zu erwartende Reaktion hinaus, kann sie als Anpassungsstörung klassifiziert werden. Nach Bengel u. Hubert [4] liegen Anpassungsstörungen im Übergangsbereich zwischen normalen angemessenen Reaktionen und spezifischen psychischen Störungen. Sie bewerten sie als die am häufigsten diagnostizierte Störung bei schweren bzw. chronischen körperlichen Erkrankungen.

Spezifische Phobie. Bei der spezifischen Phobie handelt es sich um eine ausgeprägte Furcht, die auf spezifische Situationen oder Objekte beschränkt ist. Bei der Ausbildung dieses Störungsbildes im Kontext der GUV kommt es z. B. nach Verkehrsunfällen zu Furcht beim Fahren mit Fahrzeugen und situativer Furcht im Straßenverkehr, Höhenangst nach Stürzen, Furcht an Maschinen, bei Dunkelheit, vor Personen mit bestimmter Kleidung, z. B. nach Überfällen. Phobische Reaktionen können auch vor bestimmten medizinischen Eingriffen oder vor massivem Schmerzerleben beobachtet werden.

\section{Posttraumatische Belastungsstörung} (PTBS). Die PTBS entsteht als Reaktion auf ein traumatisches Ereignis oder eine Situation außergewöhnlicher Bedrohung oder katastrophenartigen Ausmaßes, kurz oder lang anhaltend, das bei fast jedem eine tiefe Verzweiflung hervorrufen würde [7]. Ein Hauptmerkmal der PTBS ist das wiederholte Erleben des Traumas in intrusiven Erinnerungen und Flashbacks sowie in Albträumen. Zudem tritt ein Zustand vegetativer Übererregtheit mit Vigilanzsteigerung, einer übermäßigen Schreckhaftigkeit und ausgeprägten Schlafstörungen auf. Angst und Depression sind mit den genannten Symptomen und Merkmalen oft verbunden. Weiterhin typisch ist ein ausgeprägtes Vermeidungsverhalten in Bezug auf traumaassoziierte Stimuli und Situationen, aber auch Gedanken und Gefühlen zum erlebten Trauma [5]. Es kann zu emotionaler Taubheit kommen, die sich in Teilnahmslosigkeit und emotionaler Distanz äußert.

Die Störung folgt dem Trauma mit einer Latenz, die Wochen bis Monate dauern kann. Der Verlauf ist wechselhaft, in der Mehrzahl der hier diskutierten Monotraumata kann jedoch insbesondere bei konsequenter Therapieeinleitung eine Heilung erwartet werden.

Zur Häufigkeit von traumabezogenen Störungen zeigt die Untersuchung von Kühn [9] an Patienten nach schweren Unfällen, dass innerhalb der ersten Tage nach dem Unfall 7\% der Patienten eine akute Belastungsreaktion im Vollbild und $12 \%$ eine Teilsymptomatik entwickelten, 1,5\% eine Anpassungsstörung. Sechs Monate später litten $10 \%$ der untersuchten Patienten an einer Depression, $6 \%$ hatten eine PTBS im Vollbild und zusätzlich $4 \%$ eine Teilsymptomatik entwickelt; $1,5 \%$ der Patienten wiesen eine spezifische Phobie auf.

\section{Einsatzschwerpunkte von Psychotherapeuten}

Eine erste Abklärung einer sich abzeichnenden Symptomatik kann bei betroffenen Unfallversicherten zeitnah nach dem Schadensereignis erfolgen. Bereits zu diesem frühen Zeitpunkt setzt das Psychotherapeutenverfahren ein.

Am häufigsten werden Psychotherapeuten beauftragt, wenn sich in der weiteren Folge des Unfalls eine psychische Störung manifestiert oder gesetzlich Unfallversicherte Schwierigkeiten mit der Bewältigung einer persistierenden körperlichen Symptomatik (z. B. anhaltende Schmerzen, Brandnarben, Amputation) haben. Eine Therapie kann dabei auch bei subsyndromalen Störungsbildern indiziert sein, wenn sie nachvollziehbar zur Wiedererlangung der Teilhabe beitragen kann. Auch in der Begleitung der rehabilitativen Schritte bis zur Reintegration ins Erwerbsleben werden Psychotherapeuten bei besonderem Bedarf zur Unterstützung von Unfallversicherten eingebunden.

Schließlich werden Psychotherapeuten aktiv, wenn sich im Heilverfahren psychische Auffälligkeiten zeigen, die einer ausführlicheren differenzialdiagnostischen Klärung bedürfen, oder wenn unfallferne aufrechthaltende Einflüsse abgeklärt werden müssen. 


\section{Psychotraumatologi- sche Ambulanzen an BG-Unfallkliniken}

Die Psychotrauma-Ambulanzen haben eine zentrale Rolle im Netzwerk der Patientenversorgung von Unfallversicherungsträgern, Durchgangs(D)-Ärzten und ambulanten Psychotherapeuten [11].

Neben der Betreuung stationärer Patienten in den berufsgenossenschaftlichen Unfallkliniken ist die Behandlung ambulanter Patienten im Rahmen des Psychotherapeutenverfahrens ein Schwerpunkt des Angebotsspektrums. Die Frequenz der Behandlungen kann dabei in Abhängigkeit von der Indikation von intensiven Blocktherapien (z. B. Konfrontationsbehandlung bei Fahrphobie mit mehreren Therapiestunden täglich über einen kurzen Zeitraum) bis hin zu niedrigschwelligen Therapieangeboten bei schweren chronischen Verläufen variieren.

Bei schwierigen Heilverläufen, unklarer Diagnose/Kausalität und Indikation zur Psychotherapie werden umfassende Heilverfahrenskontrollen durchgeführt. Die Berichterstattung einer Heilverfahrenskontrolle umfasst klare Aussagen zur Diagnose, zum weiteren Prozedere und zur Zuständigkeit des Kostenträgers.

Bei chronifizierten psychotraumatologischen Beschwerden werden an den aktuellen AWMF-Leitlinien [10] orientierte Begutachtungen übernommen.

Die Vermittlung eines Patienten an einen Therapeuten aus dem Psychotherapeutenverfahren oder an einen psychiatrischen Kollegen sowie die Indikationsstellung für eine stationäre Therapiemaßnahme gehören ebenfalls zum Leistungsspektrum.

\section{Das „"neue" \\ Psychotherapeutenverfahren}

Für Unfallfolgen auf psychischem Fachgebiet hatte die DGUV bereits 2001 das Modellverfahren „Einbindung von ärztlichen und psychologischen Psychotherapeuten in das berufsgenossenschaftliche Heilverfahren bei psychischen Gesundheitsschäden“ eingeführt ([8], s. • Infobox). Anders als für den Bereich der chirurgisch-orthopädischen Unfallverletzungen fehlte es bis

Trauma Berufskrankh 2015 · [Suppl 2]: 17:275-280 DOI 10.1007/s10039-015-0006-8

○) Springer-Verlag Berlin Heidelberg 2015

C. Drechsel-Schlund · M. Weiß · C. Krahl · K. Romer-Raschidi • B. Gruner · H. Freytag • R. Hoffmann

\section{Umsetzung des Psychotherapeutenverfahrens. Einbindung von ärztlichen und psychologischen Psychotherapeuten}

\section{Zusammenfassung}

Hintergrund. Das Psychotherapeutenverfahren der Deutschen Gesetzlichen Unfallversicherung wurde 2012 neu definiert.

Ziel der Arbeit. In dieser Arbeit werden die Regelungen im Psychotherapeutenverfahren mit den Qualifikationsanforderungen und dem Verfahrensablauf dargestellt. Methoden. Statistiken der Unfallversicherungsträger wurden ausgewertet und diskutiert.

Ergebnisse. Mit der Einführung des Psychotherapeutenverfahrens leistet die gesetzliche Unfallversicherung ihren Beitrag zur frühzei- tigen und adäquaten Versorgung von Versicherten mit psychischen Störungen. Schlussfolgerung. Das neue Psychotherapeutenverfahren hat sich bewährt. Weitere Anstrengungen sind notwendig, um qualifizierte Rehabilitationsprozesse bei psychischen Unfallfolgen zu optimieren.

Schlüsselwörter

Unfälle · Posttraumatische

Belastungsstörungen · Unfallversicherung ·

Rehabilitation · Psychotherapie

\section{Implementation of the psychotherapist procedure. Integration of medical and psychological psychotherapists}

\section{Abstract}

Background. The procedures for integrating psychotherapy were amended by the German statutory accident insurance (Deutsche Gesetzliche Unfallversicherung, DGUV) in 2012.

Objectives. The article presents the organizational regulations including formal qualifications of psychotherapists.

Methods. The official statistics of German accident insurance companies are analyzed and discussed.

Results. With the implementation of the standardized procedure of integrating psychotherapy, the German statutory accident insurance contributes at an early stage to adequate treatment of insured persons with mental health problems.

Conclusion. The new procedure for integrating psychotherapy into medical treatment has proved its value. More efforts are necessary to extend qualified rehabilitation of patients with mental disorders in the framework of statutory accident insurance.

\section{Keywords}

Accidents · Posttraumatic stress disorders . Accident insurance $\cdot$ Rehabilitation .

Psychotherapy dahin an entsprechenden Versorgungsstrukturen und besonderen Verfahren für die Heilbehandlung. Das Modellverfahren wurde zum 01.07.2012 durch das neue Psychotherapeutenverfahren [6] abgelöst. Bei der grundlegenden Überarbeitung der Regelungen haben aktuelle wissenschaftlich-medizinische Erkenntnisse zur Diagnostik und Therapie von psychischen Störungen, die Erfahrungen der Unfallversicherungs(UV)-Träger sowie Ergebnisse von Expertengesprächen mit Vertretern der relevanten wissenschaftlich-medizinischen Fachgesellschaften sowie der Verbände bzw. der Standesorganisationen Berücksichtigung gefunden.
Das Psychotherapeutenverfahren soll die Versorgung von der Akutintervention bis zur beruflichen Reintegration sicherstellen und alle Beteiligten einbinden: die Versicherten, die behandelnden Psychotherapeuten, die jeweiligen Arbeitgeber, die D-Ärzte und die UV-Träger. Ziel ist, dass Unfallversicherte mit psychischen Auffälligkeiten bzw. Störungen frühzeitig und adäquat professionelle Hilfe erhalten. Die UV-Träger und auch die DÄrzte sind als Lotsen bei der Heilverfahrenssteuerung gefordert, gefährdete Versicherte zu identifizieren, um einen zeitnahen Zugang zum Psychotherapeutenverfahren zu ermöglichen. 


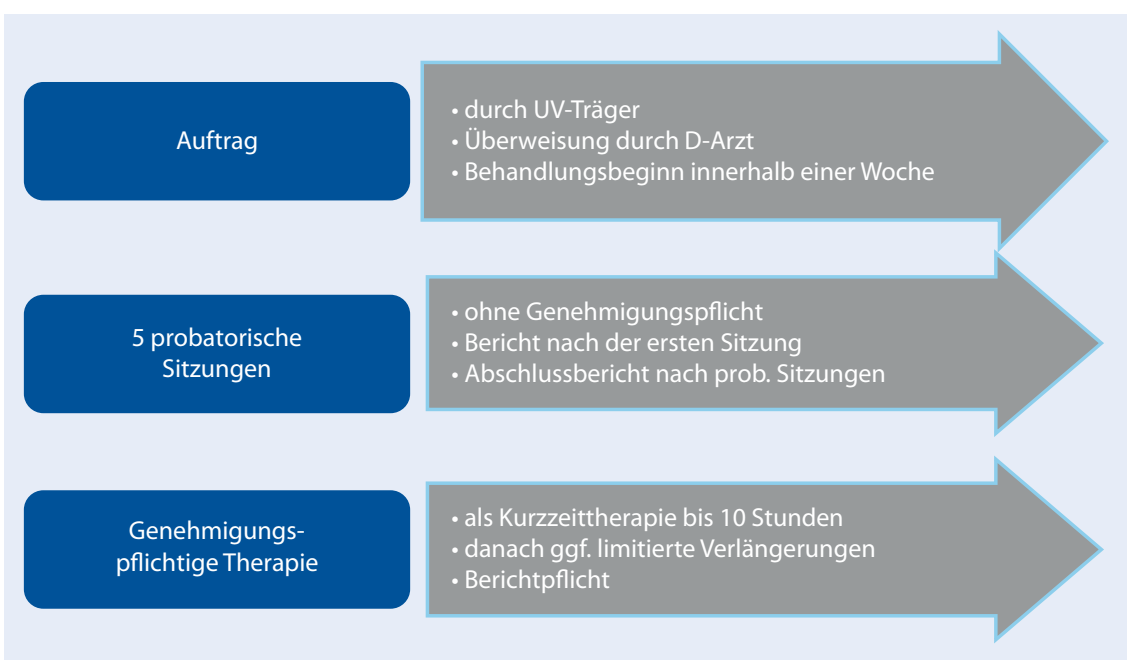

Abb. $2 \Delta$ Ablauf des Psychotherapeutenverfahrens

\section{Anforderungen und Handlungsanleitung}

Das neue Psychotherapeutenverfahren besteht aus zwei Regelungselementen: den Anforderungen und einer Handlungsanleitung. Die Anforderungen zum Psychotherapeutenverfahren enthalten die notwendigen fachlichen Qualifikationsvoraussetzungen und weitere Bestimmungen für die Zulassung von Leistungserbringern. In der Handlungsanleitung zum Psychotherapeutenverfahren werden der generelle Ablauf im Behandlungsprozess und die Zusammenarbeit mit den UVTrägern normiert.

Die Anforderungen sehen vor, dass psychologische Psychotherapeuten mit Approbation in einer der drei anerkannten Richtlinienverfahren sowie psychotherapeutisch tätige Fachärzte als Leistungserbringer beteiligt werden können, wenn sie zusätzlich über Fachkenntnisse in der leitliniengerechten Diagnostik und Behandlung von typischen Störungen nach Arbeitsunfällen und Berufskrankheiten verfügen. Die Psychotherapeuten müssen eine bestimmte Zahl von spezifischen Behandlungsfällen vorweisen, evidenzbasierte Behandlungsverfahren anwenden und sich an den Leitlinien der Arbeitsgemeinschaft der Wissenschaftlich-Medizinischen Fachgesellschaften (AWMF) orientieren, insbesondere an der Leitlinie „Akute Folgen psychischer Traumatisierung - Diagnostik und Behandlung", die derzeit überprüft wird [3]. Darüber hinaus haben sie beson- dere Pflichten im Rahmen des SGB-VIIVersorgungsauftrags zu übernehmen. So müssen die Psychotherapeuten beispielsweise das Reha-Management der UV-Träger unterstützen und den Fokus auf eine schnelle berufliche Reintegration richten.

Durch die Handlungsanleitung wird eine einheitliche Umsetzung erreicht. Von den Netzwerktherapeuten wird verlangt, nach SGB VII Versicherte innerhalb einer Woche in die Behandlung zu übernehmen. Die Sitzungsfrequenz ist individuell festzulegen. Sitzungen sollen jedoch regelmäßig im Abstand von 1 Woche bis maximal 2 Wochen stattfinden. Die beteiligten Psychotherapeuten haben den UV-Trägern kontinuierlich über den Behandlungsverlauf zu berichten. Auf diese Weise kann eine zielgerichtete, aktive Heilverfahrenssteuerung (Reha-Management) auch bei psychischen Unfallfolgen sichergestellt werden.

Die Netzwerk-Psychotherapeuten bzw. am Psychotherapeutenverfahren beteiligten Leistungserbringer können über den Internetauftritt der Landesverbände der DGUV mit Kontaktdaten recherchiert werden. Qualifizierte Psychotherapeuten können zudem bei allen UV-Trägern oder bei den Landesverbänden der DGUV erfragt werden.

\section{Ablauf im Behandlungsfall}

Die effiziente und effektive Behandlung psychischer Störungen erfordert ein frühzeitiges Einsetzen geeigneter Maßnahmen. Daher sind Betroffene mit psychi- schen Symptomen, etwa Schlafstörungen oder Ängsten, rasch einem Netzwerk-Psychotherapeuten vorzustellen. Hierdurch wird eine schnelle diagnostische Abklärung ermöglicht. Sowohl der UV-Träger als auch der D-Arzt können die Konsultation eines Psychotherapeuten bzw. die Einleitung probatorischer Sitzungen initiieren (•Abb. 2). Unfallversicherte erhalten unbürokratisch bis zu 5 probatorische Sitzungen, ohne dass eine Genehmigung des UV-Trägers benötigt wird. Im Interesse der Frühintervention wird im Rahmen der probatorischen Sitzungen die an sich notwendige Beurteilung, ob die psychischen Störungen unfallbedingt sind, zunächst zurückgestellt. Weitere Sitzungen bedürfen dann jedoch einer Antragstellung durch den Therapeuten und einer Genehmigung durch den UV-Träger. Die Genehmigung erfolgt regelmäßig für zunächst maximal 10 Sitzungen. Um „Behandlungslöcher" zu vermeiden, sind die Anträge auf Weiterbehandlung rechtzeitig zu stellen und vom UV-Träger zeitnah zu entscheiden.

\section{Die Rolle des D-Arztes}

Hinweise auf psychische Symptome zeigen sich für den D-Arzt oftmals bereits bei der Erstvorstellung, wenn Versicherte über das Unfallerlebnis und ihre Beschwerden berichten. In einigen Fällen werden Angaben zu einer psychischen Belastung von den Betroffenen erst bei der Wiedervorstellung gemacht oder deutlicher erkennbar. Im Zwischenbericht wird der D-Arzt bei besonderer Heilbehandlung ausdrücklich aufgefordert, entsprechende Hinweise auf psychische Störungen darzulegen und den UV-Träger $\mathrm{zu}$ informieren. So wie andere Fachärzte zur Mitbehandlung hinzugezogen werden, kann der D-Arzt die Vorstellung bei einem Netzwerk-Psychotherapeuten auch selbst veranlassen.

Über den UV-Träger wird sichergestellt, dass der D-Arzt Konsilberichte der mitbehandelnden Psychotherapeuten erhält. Bei der Behandlung durch psychologische Psychotherapeuten greift die Auskunftspflicht nach $₫ 201$ SGB VII (Datenerhebung und -verarbeitung durch Ärzte) nicht. Der UV-Träger verbindet den Behandlungsauftrag daher stets mit einer 


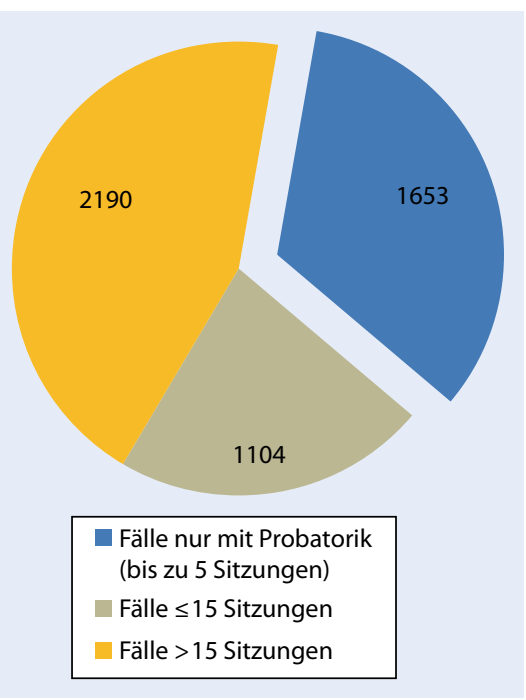

Abb. $3 \Delta$ Statistische Daten zum Psychotherapeutenverfahren 2013

schriftlichen Einwilligungserklärung zur Weitergabe der Daten, auch für die Information des behandelnden D-Arztes.

Es ist Aufgabe des qualifizierten Psychotherapeuten, eine Diagnose auf psychischem Fachgebiet zu klären bzw. zu sichern. Insbesondere die häufig komplexe Differenzialdiagnostik erfordert fachliche Expertise. Im D-Arzt-Bericht sollten daher einschlägige "Verdachtsdiagnosen“ (z. B. Verdacht auf eine PTBS) vermieden werden. Vielmehr ist ausreichend, wenn aus dem D-Arzt Bericht hervorgeht, dass auffällige psychische Beschwerden bzw. Symptome vorliegen und es einer Konsultation zur Diagnosevalidierung bedarf (Beispiele für Formulierungen: „psychische Belastung nach Unfall“ oder "psychoreaktive Störungslage nach Unfallgeschehen").

\section{Dialog mit \\ Netzwerk-Psychotherapeuten}

Für die Beteiligung im Psychotherapeutenverfahren ist die Teilnahme an einer Einführungsveranstaltung obligatorisch. In den Jahren 2013 und 2014 haben die Landesverbände der DGUV insgesamt 9 Einführungsveranstaltungen mit über 600 Teilnehmern durchgeführt. Auf dem Programm standen insbesondere die speziellen Rahmenbedingungen der GUV, die sich in Bezug auf das Kausalitätserfordernis und die Heilverfahrenssteuerung ganz wesentlich von denen der gesetzli- chen Krankenversicherung unterscheiden. Bei den Einführungsveranstaltungen haben regelmäßig auch Vertreter der Psychotrauma-Ambulanzen der BG-Unfallklinken und der UV-Träger mitgewirkt. Auf diese Weise war es möglich, in einen intensiven Dialog mit den Netzwerk-Psychotherapeuten einzutreten.

\section{Zahlen und Daten}

Am Psychotherapeutenverfahren der DGUV waren mit Stand 08.10.2014 insgesamt 496 Netzwerkpartner beteiligt. Im Rahmen dieses Verfahrens wurden im Jahr 2013 insgesamt 5139 Behandlungsfälle gemeldet. Diese Zahlen bilden nicht alle Behandlungsfälle mit probatorischen Sitzungen bzw. Psychotherapie ab, weil die UV-Träger teilweise auf eigene Therapeutenlisten zurückgreifen und die statischen Angaben z. T. unvollständig sind (Rücklaufquote $93,5 \%$ ). Im Verhältnis zu den ca. 1,06 Mio. meldepflichtigen Arbeitsunfällen 2013 stellt die Anzahl der Behandlungsfälle im Psychotherapeutenverfahren eine kleine Fallmenge dar. Die Dauer der Behandlung zeigt eine Streuung, wobei ein Behandlungsabschluss in 33\% der Fälle mit 5 probatorischen Sitzungen und kumuliert in einem Anteil von 55\% nach weiteren 10 Sitzungen der Weiterbehandlung erreicht werden konnte (insgesamt 4947 auswertbare Behandlungsfälle, - Abb. 3).

Aus den statistischen Angaben der Netzwerk-Psychotherapeuten wird deutlich, dass die Versicherten der GUV nur einen untergeordneten Anteil der Patienten im Praxisbetrieb ausmachen. Im Jahr 2013 waren es für jeden Netzwerkpartner im Mittelwert 12 Versicherte der UV-Träger. Das Versorgungsnetz erscheint im Hinblick auf die Relation von Behandlungsfällen und Behandlern gut entwickelt, unter Berücksichtigung der kurzen Zeitspanne seit der Einführung des Psychotherapeutenverfahrens Mitte 2012. In einigen Regionen der neuen Bundesländer und in ländlichen Gebieten sind die Versorgungsstrukturen aber noch nicht ausreichend. Dies ist bei psychischen Unfallfolgen besonders problematisch, wenn Unfallversicherte nicht wohnortnah behandelt werden können. Hier müssen mit allen geeigneten Mitteln Lösungen gefunden werden.

\section{Fazit für die Praxis}

- Ziele des Psychotherapeutenverfahrens sind die frühzeitige und adäquate Versorgung von Versicherten mit unfallbedingten psychischen Störungen.

- Das Kooperationsnetzwerk ist interdisziplinär ausgerichtet und umfasst ambulante Psychotherapeuten, Durchgangsärzte, UV-Träger und Psychotrauma-Ambulanzen an den Unfallkliniken.

- Weitere Anstrengungen sind notwendig, um qualifizierte Rehabilitationsprozesse von der Frühintervention bis hin zur beruflichen Wiedereingliederung auch bei psychischen Unfallfolgen flächendeckend sicherstellen zu können.

\section{Korrespondenzadresse

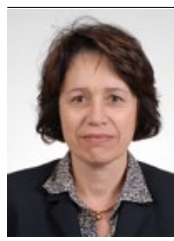 \\ C. Drechsel-Schlund Berufsgenossenschaft für Gesundheitsdienst und Wohlfahrtspflege Bezirksverwaltung Würzburg Röntgenring 2 97070 Würzburg Claudia.Drechsel-Schlund@ bgw-online.de}

\section{Einhaltung ethischer Richtlinien}

Interessenkonflikt. C. Drechsel-Schlund, M. Weiß, C. Krahl, K. Romer-Raschidi, B. Gruner, H. Freytag und R. Hoffmann geben an, dass kein Interessenkonflikt besteht.

Dieser Beitrag beinhaltet keine Studien an Menschen oder Tieren.

The supplement containing this article is not sponsored by industry.

\section{Literatur}

1. Angenendt J (2012) Psychische Störungen nach Gewalterleben und Bedrohung - Einführung aus medizinisch-psychologischer Sicht. MedSach 108(3):106-110

2. Angenendt J, Riering A, Röhrich B, Südkamp N, Berger M (2012) Freiburger Arbeitsunfallstudie-II (FAUST-II). Trauma Berufskrankh 14(Suppl 3):299_ 306 
3. AWMF (2014) Geplante Leitlinie "Akute Folgen psychischer Traumatisierung - Diagnostik und Behandlung". AWMF-Register Nr. 051/027: http:// www.awmf.org/leitlinien/detail/anmeldung/1/ II/051-027.html. Zugegriffen: 07. Jan. 2015

4. Bengel J, Hubert S (2010) Anpassungsstörung und akute Belastungsstörung. Hogrefe, Göttingen

5. Boos A, Müller J (2006) Posttraumatische Belastungsstörungen. In: Wittchen HU, Hoyer J (Hrsg) Klinische Psychologie \& Psychotherapie. Springer, Berlin Heidelberg New York

6. DGUV-Rundschreiben - 0284/2012 vom 27.06.2012 (unveröffentlicht)

7. Dilling H, Mombour W, Schmidt MH (Hrsg) (2008) Taschenführer zur ICD-10-Klassifikation psychischer Störungen. Huber, Bern

8. Drechsel-Schlund C (2004) Arbeitsunfall und psychische Gesundheitsschäden - Modellverfahren aus der Sicht der Unfallversicherungsträger. Trauma und Berufskrankh 6(Suppl 1):S46-S50

9. Kühn M, Ehlert U, Rumpf HJ, Backhaus J, Hohagen F, Brooks A (2006) Onset and maintenance of psychiatric disorders after serious accidents. Eur Arch Psychiatry Clin Neurosci 256(8):497-503

10. Schneider W, Henningsen P, Dohrenbusch R, Freyberger HJ, Irle H, Köllner V, Widder B (2012) Begutachtung bei psychischen und psychosomatischen Erkrankungen. Autorisierte Leitlinien und Kommentare. Huber, Bern

11. Schulz B, Ullmann U (2007) Psychotraumatologische Versorgung im bg-lichen Heilverfahren. Trauma und Berufskrankh 2007(Suppl 1):109-112

12. Wind G, Romer-Raschidi K, Stolle A, Ripper S, Kühn C (2010) Psychotraumatologie. In: Wölfl C, Matthes $\mathrm{G}$ (Hrsg) Unfallrettung. Schattauer, Stuttgart 